\title{
Analysis of Mismatch Effects in Randomly Interleaved A/D Converter System
}

\author{
Jonas Elbornsson, Fredrik Gustafsson \\ Jan-Erik Eklund \\ Control \& Communication \\ Department of Electrical Engineering \\ Linköpings universitet, SE-581 83 Linköping, Sweden \\ WWW: http://www. control.isy.liu.se \\ E-mail: jonas@isy.liu.se, fredrik@isy.liu.se
}

3rd March 2003

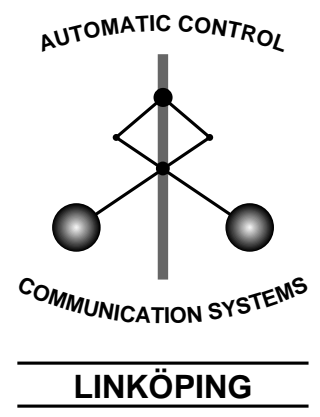

Report no.: LiTH-ISY-R-2496

Submitted to

Technical reports from the Control \& Communication group in Linköping are available at http://www. control.isy.liu.se/publications. 


\begin{abstract}
To significantly increase the sampling rate of an A/D converter (ADC), a time interleaved ADC system is a good option. The drawback of a time interleaved ADC system is that the ADCs are not exactly identical due to errors in the manufacturing process. This means that time, gain and offset mismatch errors are introduced in the ADC system. These errors cause non harmonic distortion in the sampled signal.

One way to decrease the impact of the mismatch errors is to spread the distortion over a wider frequency range by randomizing the order in which the ADCs are used in the interleaved structure. In this paper we analyze how the spectrum is affected by mismatch errors in a randomly interleaved ADC system. We also discuss how the mismatch errors can be estimated.
\end{abstract}

Keywords: A/D conversion, nonuniform sampling, random sampling 


\title{
Analysis of Mismatch Effects in Randomly Interleaved A/D Converter System
}

\author{
J. Elbornsson, J.-E. Eklund, F. Gustafsson
}

\begin{abstract}
To significantly increase the sampling rate of an A/D converter (ADC), a time interleaved ADC system is a good option. The drawback of a time interleaved ADC system is that the ADCs are not exactly identical due to errors in the manufacturing process. This means that time, gain and offset mismatch errors are introduced in the ADC system. These errors cause non harmonic distortion in the sampled signal.

One way to decrease the impact of the mismatch errors is to spread the distortion over a wider frequency range by randomizing the order in which the ADCs are used in the interleaved structure. In this paper we analyze how the spectrum is affected by mismatch errors in a randomly interleaved ADC system. We also discuss how the mismatch errors can be estimated.
\end{abstract}

Index Terms- A/D conversion, nonuniform sampling, random sampling

\section{INTRODUCTION}

The requirements for higher sample rates in A/D converters (ADCs) are ever increasing. To achieve high enough sample rates, a time interleaved ADC system can be used [1], [2], see Fig. 1. The time interleaved ADC system works as follows:

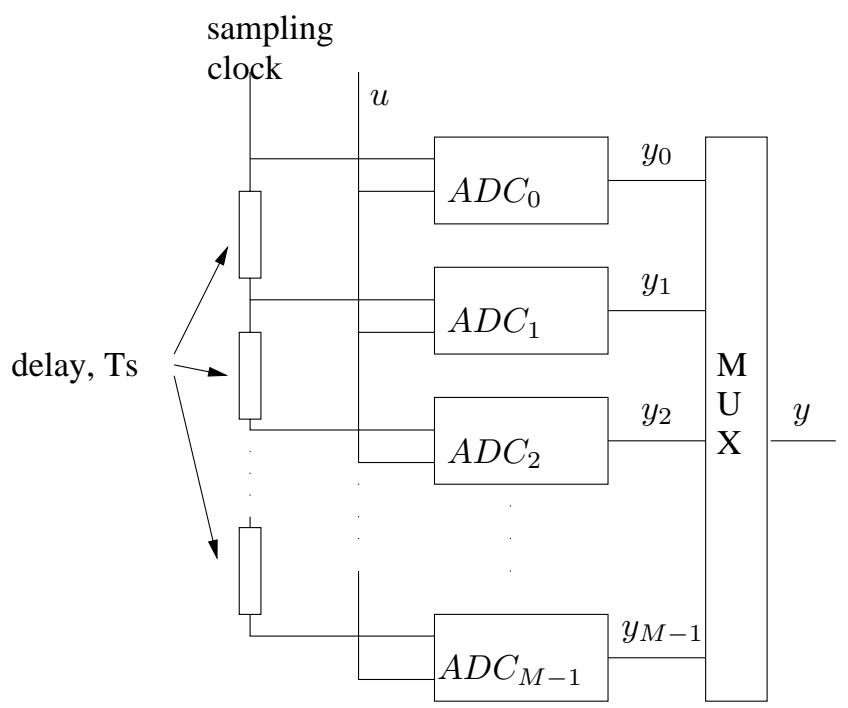

Fig. 1. A time interleaved ADC system. $M$ parallel ADCs are used with the same master clock. The clock is delayed by the nominal sampling interval to each ADC. The outputs are then multiplexed together to form a signal sampled $M$ times faster than the output from each ADC.

- The input signal, $u$, is connected to all the ADCs.

- Each ADC works with a sampling interval of $M T_{s}$, where $M$ is the number of ADCs in the array and $T_{s}$ is the desired sampling interval. The $i$ th ADC gives an output signal $y_{i}$. The output signals are multiplexed to form one output signal $y$.
- The clock signal to the $i$ th ADC is delayed with $i T_{s}$. This gives an overall sampling interval of $T_{s}$.

The drawback with this ADC system is that three kinds of mismatch errors are introduced by the interleaved structure:

- Time errors (static jitter)

The delay time of the clock to the different A/D converters is not equal. This means that the signal will be periodically but non-uniformly sampled.

- Amplitude offset errors

The ground level can be slightly different in the different $\mathrm{A} / \mathrm{D}$ converters. This means that there is a constant amplitude offset in each A/D converter.

\section{- Gain error}

The gain, from analog input to digital output, can be different for the different A/D converters.

All these errors distort the sampled signal. With a sinusoidal input, the mismatch errors can be seen in the output spectrum as non harmonic distortion. With input signal frequency $\omega_{0}$, the gain and time errors cause distortion at the frequencies

$$
\frac{i}{M} \omega_{s} \pm \omega_{0}, i=1, \ldots, M-1,
$$

where $\omega_{s}$ is the sampling frequency. The offset errors cause distortion at the frequencies

$$
\frac{i}{M} \omega_{s}, i=1, \ldots, M-1 .
$$

An example of an output spectrum from an interleaved ADC system with four ADCs with sinusoidal input signal is shown in Fig. 2. This distortion causes problems for instance in a radio receiver where a weak carrier cannot be distinguished from the mismatch distortion from a strong carrier. It is therefore important to minimize the impact of the distortion.

\section{A. Random interleaving}

One way to decrease the impact of the distortion is to randomize the selection of which ADC that should be used at each time instance. This means that an ADC is picked at random at each sampling instance. However, the reason for using the interleaved structure is that each $\mathrm{A} / \mathrm{D}$ converter needs $M$ times the desired sampling rate to complete the sampling. Therefore only one ADC is available for selection at each sampling instance. However, to achieve some randomization one or more extra ADCs can be used [3], see Fig. 3. With $\Delta M$ additional ADCs there are always $\Delta M+1$ ADCs available to select from at each sampling instance. An example of the possible ADC selections for $M=4$ and $\Delta M=1$ is shown in Fig. 4. The randomization spreads the spikes in the spectrum to a more noise-like shape. The spectrum for this kind of ADC system will be calculated in detail in Section III. 


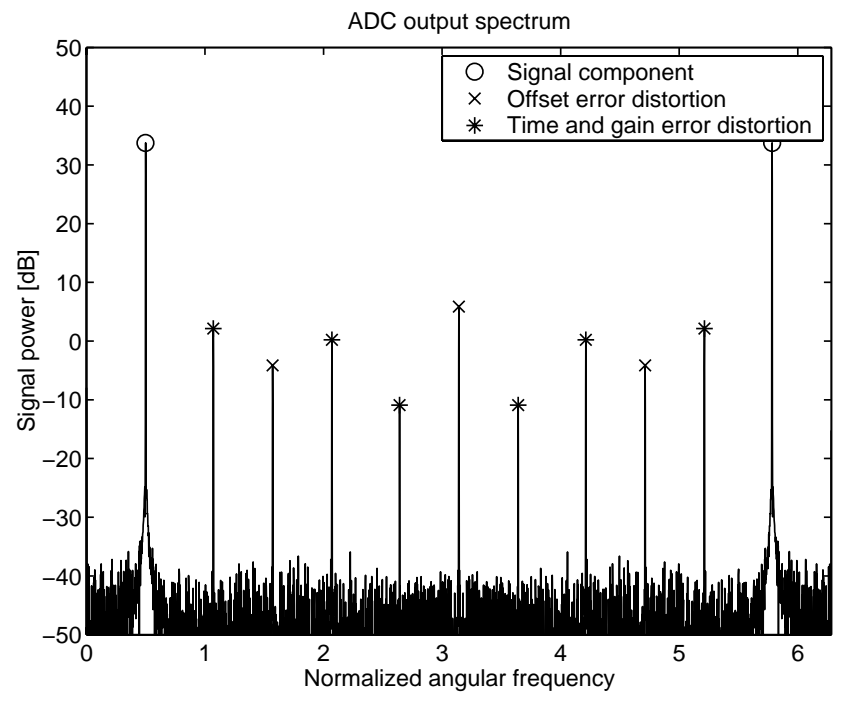

Fig. 2. Simulated output spectrum from interleaved ADC system with four ADCs. The input signal is a single sinusoid. The distortion is caused by mismatch errors.

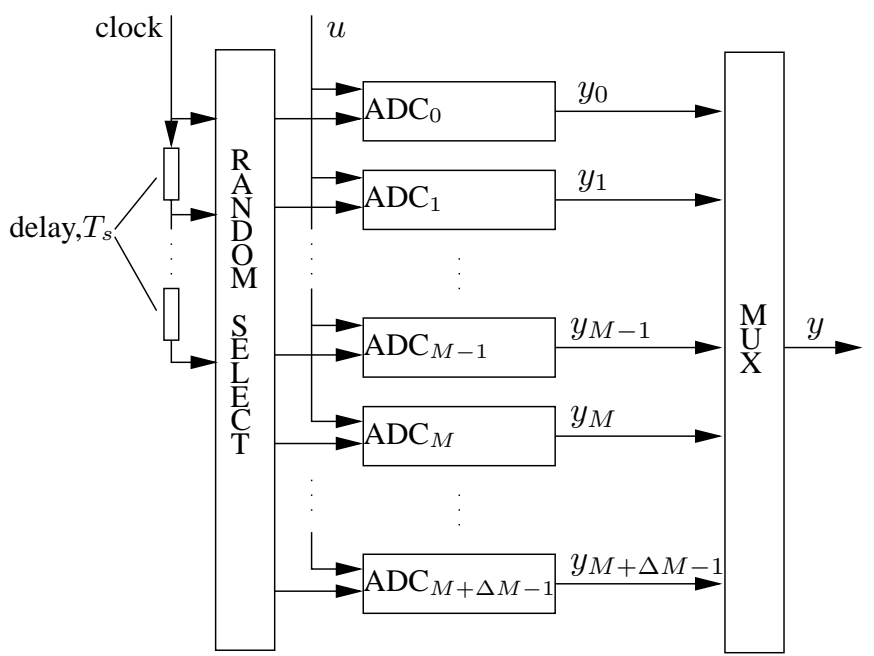

Fig. 3. Random interleaved ADC system with $M$ times higher sampling rate than in each ADC. $\triangle M$ additional $\mathrm{ADCs}$ are used to achieve some randomization, i.e., $\Delta M+1 \mathrm{ADCs}$ are available at each sampling instance.

\section{Notations AND DEFINITIONS}

In this section we introduce the notations used in the rest of the paper. We assume throughout the rest of the paper that the overall sampling interval, for the complete ADC system, is one. This assumption is done to simplify notation and is no restriction.

We denote by $M$ the number of ADCs required to achieve the desired sampling rate, where each ADC needs the time $M T_{s}$ to complete a conversion. $\Delta M$ denotes the number of additional ADCs used to randomize the spectrum. The total number of ADCs in the system are $M+\Delta M$. The time, gain and offset errors are denoted $\Delta_{t, i}^{0}, \Delta_{g, i}^{0}, \Delta_{o, i}^{0}, i=$ $0, \ldots, M-1, M, \ldots, M-1+\Delta M$ respectively. The sampling time instances for each ADC are picked at random and $X_{k}$ denotes the ADC used at time $k$. The time instances when the

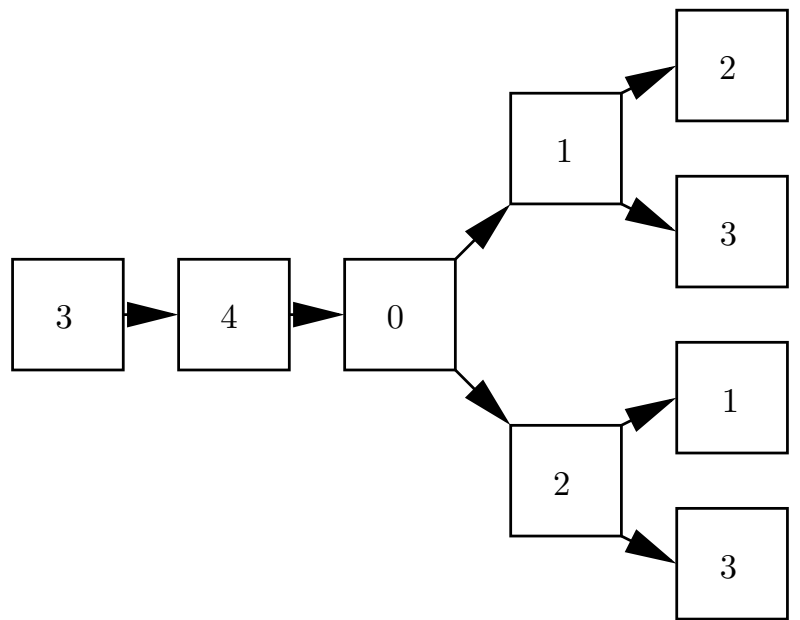

Fig. 4. An example of the possible ADC selection order for $M=4$ and $\Delta M=1$.

$i$ th ADC is used are denoted $k_{i}$. We use the following notation for the signals involved:

- $u(t)$ is the analog input signal.

- $u[k]$ is the input signal, sampled without errors.

- $y_{i}\left[k_{i}\right]$ are the output subsequences from the $M+\Delta M$ ADCs.

$$
\begin{aligned}
& y_{i}\left[k_{i}\right]=\left(1+\Delta_{g, i}^{0}\right) u\left(k_{i}+\Delta_{t, i}^{0}\right)+\Delta_{o, i}^{0}+e_{q}\left[k_{i}\right] \\
& i=0,1, \ldots, M+\Delta M-1 .
\end{aligned}
$$

Here $e_{q}[k]$ is quantization noise. The quantization noise is assumed to be uniformly distributed and white.

- $y[k]$ is the multiplexed output signal from the randomized subsequences from all the ADCs. The subsequences are multiplexed together to form a signal with correct time ordering. The output signal can be expressed by

$$
y[k]=\left(1+\Delta_{g, X_{k}}^{0}\right) u\left(k+\Delta_{t, X_{k}}^{0}\right)+\Delta_{o, X_{k}}^{0}+e_{q}[k] .
$$

We assume throughout this paper that $u(t)$ is band limited to the Nyquist frequency.

We will next establish a few definitions which will be used later in the paper. A discrete time signal $u[k]$ is said to be quasi-stationary [4] if

$$
\begin{aligned}
& \bar{m}_{u}=\lim _{N \rightarrow \infty} \frac{1}{N} \sum_{k=1}^{N} E\{u[k]\} \\
& \bar{R}_{u}[n]=\lim _{N \rightarrow \infty} \frac{1}{N} \sum_{n=1}^{N} E\{u[k+n] u[k]\}
\end{aligned}
$$

exist, where the expectation is taken over possible stochastic parts of the signal. Analogously, a continuous time signal $u(t)$ is quasi-stationary if

$$
\begin{aligned}
& \bar{m}_{u}=\lim _{T \rightarrow \infty} \frac{1}{T} \int_{0}^{T} E\{u(t)\} d t \\
& \bar{R}_{u}(\tau)=\lim _{T \rightarrow \infty} \frac{1}{T} \int_{0}^{T} E\{u(t+\tau) u(t)\} d t
\end{aligned}
$$


exist. A stationary stochastic process is quasi-stationary, with $\bar{m}_{u}$ and $\bar{R}_{u}[n]$ being the mean value and covariance function respectively. Assume that $u[k]$ is quasi-stationary. Then the power spectrum of $u[k]$ is defined as [4]:

$$
\Phi_{u}\left(e^{i \omega}\right)=\sum_{n=-\infty}^{\infty} R_{u}[n] e^{-j \omega n} .
$$

Analogously, we define the power spectrum for continuous time signals as

$$
\Phi_{u}(\omega)=\int_{-\infty}^{\infty} R_{u}(\tau) e^{-j \omega \tau} d \tau .
$$

We will next define two concepts for measuring the performance of an ADC. Assume that the output $y[k]$ of an ADC consists of a signal part $s[k]$, a distortion part $d[k]$, and a noise part $e[k]$

$$
y[k]=s[k]+d[k]+e[k] .
$$

Then the SNDR (Signal to Noise and Distortion Ratio) is defined as

$$
S N D R=10 \log _{10}\left(\frac{E\left\{s^{2}[k]\right\}}{E\left\{d^{2}[k]\right\}+E\left\{e^{2}[k]\right\}}\right) .
$$

The SFDR (Spurious Free Dynamic Range) is defined for a sinusoidal input signal as the distance between the signal component in the spectrum and the strongest distortion component, measured in $\mathrm{dB}$, see Fig. 5.

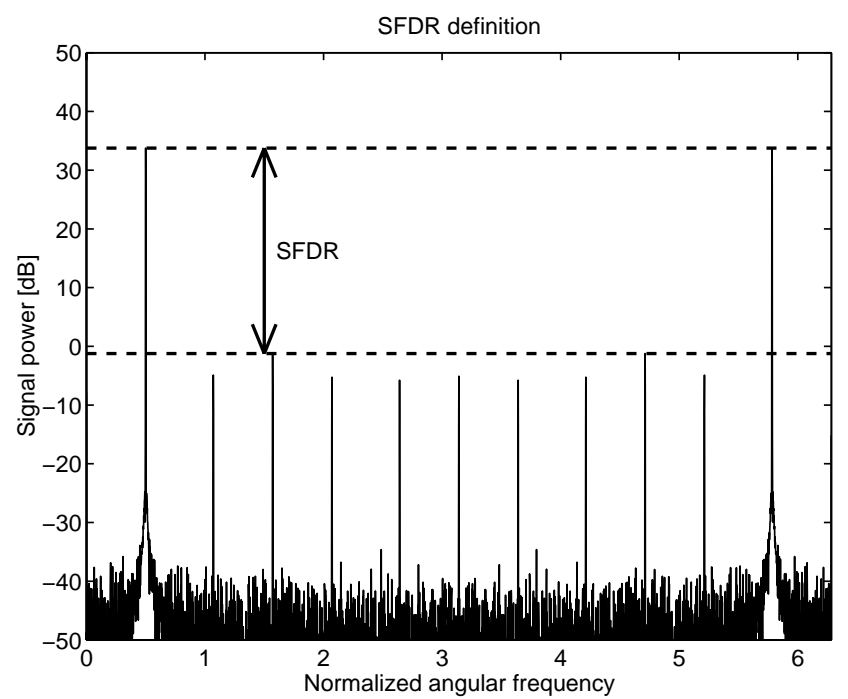

Fig. 5. The SFDR is defined for a sinusoidal input. SFDR is the difference between the signal component and the strongest distortion component, measured in $\mathrm{dB}$.

\section{MismatCH NOISE SPECTRUM}

In this section we will calculate the spectrum for the noise introduced by mismatch errors in a randomly interleaved ADC system. We will also compare the spectrum with what we get with interleaving but without randomization.
The spectrum for $y[k]$ is given by (7) as

$$
\Phi_{y}\left(e^{i \omega}\right)=\sum_{n=-\infty}^{\infty} R_{y}[n] e^{-i \omega n} .
$$

To calculate the spectrum we need the covariance function (4) for $y[k]$

$$
\begin{aligned}
& \bar{R}_{y}[n]=\lim _{N \rightarrow \infty} \frac{1}{N} \sum_{n=1}^{N} E(y[k+n] y[k]) \\
& =\lim _{N \rightarrow \infty} \frac{1}{N} \sum_{n=1}^{N} E\left\{\left[\left(1+\Delta_{g, X_{k+n}}^{0}\right) u\left(k+n+\Delta_{t, X_{k+n}}^{0}\right)\right.\right. \\
& \left.+\Delta_{o, X_{k+n}}^{0}+e_{q}[k+n]\right] . \\
& \left.\left[\left(1+\Delta_{g, X_{k}}^{0}\right) u\left(k+\Delta_{t, X_{k}}^{0}\right)+\Delta_{o, X_{k}}^{0}+e_{q}[k]\right]\right\} \\
& =E\left\{\left(1+\Delta_{g, X_{k+n}}^{0}\right)\left(1+\Delta_{g, X_{k}}^{0}\right)\right\} . \\
& \lim _{N \rightarrow \infty} \frac{1}{N} \sum_{n=1}^{N} E\left\{u\left(k+n+\Delta_{t, X_{k+n}}^{0}\right) u\left(k+\Delta_{t, X_{k}}^{0}\right)\right\} \\
& +E\left\{\Delta_{o, X_{k+n}}^{0} \Delta_{o, X_{k}}^{0}\right\}+E\left\{e_{q}[k+n] e_{q}[k]\right\} .
\end{aligned}
$$

In the last equality we have assumed that $\bar{m}_{u}=0$ for notational simplicity. However, this is no restriction, since a mean value different from zero just gives an additive constant. We have also assumed that the gain errors are independent of the input signal, which is a reasonable assumption since the samples are picked at random. We introduce the following notation for the respective parts of the last expression in (10)

$$
\begin{aligned}
& R_{\Delta_{g}}[n]=E\left\{\left(1+\Delta_{g, X_{k+n}}^{0}\right)\left(1+\Delta_{g, X_{k}}^{0}\right)\right\} . \\
& \bar{R}_{u, \Delta_{t}}[n]= \\
& \lim _{N \rightarrow \infty} \frac{1}{N} \sum_{n=1}^{N} E\left\{u\left(k+n+\Delta_{t, X_{k+n}}^{0}\right) u\left(k+\Delta_{t, X_{k}}^{0}\right)\right\} \\
& R_{\Delta_{o}}[n]=E\left\{\Delta_{o, X_{k+n}}^{0} \Delta_{o, X_{k}}^{0}\right\} \\
& R_{q}[n]=E\left\{e_{q}[k+n] e_{q}[k]\right\} .
\end{aligned}
$$

Using the above notation we can write the covariance for $y[k]$ as

$$
\bar{R}_{y}[n]=R_{\Delta_{g}}[n] \bar{R}_{u, \Delta_{t}}[n]+R_{\Delta_{o}}[n]+R_{q}[n] .
$$

Each expression above will be evaluated separately in the following sections. However, to evaluate these expressions we need a probabilistic model of the ADC system. This will be investigated next.

\section{A. Probabilistic model}

We will now study the random interleaved ADC system, as described in Fig. 3, from a probabilistic viewpoint. We have the notation $X_{k}$ for the ADC that is used to convert the signal at time $k$. Numbering the ADCs from 0 to $M+\Delta M-1$, we have that $X_{k} \in\{0, \ldots, M+\Delta M-1\}$. For the calculation 
of the covariance function in the next subsection we will need the probability that the same ADC is used at a certain time distance, $n$. We denote this probability by $P\left(X_{k+n}=X_{k}\right)$. Since each ADC needs the time $M$ to complete the conversion, the probability $P\left(X_{k+n}=X_{k}\right)$ depends on the previous $M-1$ time instances. Therefore, to calculate this probability, we first calculate the joint probability over $M-1$ time instances. To calculate this probability we introduce $2^{M-1}$ states, represented by binary sequences of length $M-1$, that the ADC system can be in at time $k+n$ :

$$
\begin{aligned}
00 \ldots 0= & \left\{X_{k+n} \neq X_{k}, X_{k+n-1} \neq X_{k}, \ldots,\right. \\
& \left.X_{k+n-(M-2)} \neq X_{k}\right\} \\
00 \ldots 1= & \left\{X_{k+n} \neq X_{k}, X_{k+n-1} \neq X_{k}, \ldots,\right. \\
& \left.X_{k+n-(M-2)}=X_{k}\right\} \\
& \vdots \\
11 \ldots 1= & \left\{X_{k+n}=X_{k}, X_{k+n-1}=X_{k}, \ldots,\right. \\
& \left.X_{k+n-(M-2)}=X_{k}\right\} .
\end{aligned}
$$

Here a 0 denotes $\neq$ and a 1 denotes $=$. Since the same ADC cannot be used within a time interval of $M-1$, most of these states are illegal.

$$
a_{1} a_{2} \ldots a_{M-1} \text {, is illegal if } a_{i}=a_{j}=1, i \neq j
$$

Removing the illegal states we have $M$ states remaining

$$
\begin{aligned}
& 10 \ldots 0 \\
& 01 \ldots 0 \\
& \vdots \\
& 00 \ldots 1 \\
& 00 \ldots 0 .
\end{aligned}
$$

We first assume that $n \geq M-1$. The joint probabilities are denoted as follows:

$$
\begin{aligned}
& P_{10 \ldots 0}^{(n)}=P(10 \ldots 0 \text { at time } k+n) \\
& P_{01 \ldots 0}^{(n)}=P(01 \ldots 0 \text { at time } k+n) \\
& \vdots \\
& P_{00 \ldots 0}^{(n)}=P(00 \ldots 0 \text { at time } k+n)
\end{aligned}
$$

and the probability state vector is denoted

$$
P^{(n)}=\left[\begin{array}{c}
P_{10 \ldots 0}^{(n)} \\
P_{01 \ldots 0}^{(n)} \\
\vdots \\
P_{00 \ldots 1}^{(n)} \\
P_{00 \ldots 0}^{(n)}
\end{array}\right] .
$$

These probabilities can be calculated recursively, and we have to treat three cases separately here:

- $P_{10 \ldots 0}^{(n)}$ :

If we step back one time instance to time $n-1$, we have the possible states $00 \ldots 0$ and $00 \ldots 1$. However, the probability of going from $00 \ldots 1$ to $10 \ldots 0$ is zero since the time distance between the use of the same ADC is $M-1$ here. This leaves the only possible previous state, $00 \ldots 0$, and since there are $1+\Delta M$ ADCs available at each time instance and the probability for selecting any of those is equal the probability of going to the state $10 \ldots 0$ is $\frac{1}{1+\Delta M}$, i.e.,

$$
P_{10 \ldots 0}^{(n)}=\frac{1}{1+\Delta M} P_{00 \ldots 0}^{(n-1)}
$$

- $P_{0 \ldots 010 \ldots 0}^{(n)}$ :

Here we have the two possible states $0 \ldots 100 \ldots 0$ and $0 \ldots 100 \ldots 1$ from the time instance before, of which only the first state is legal. Since the only possible transition from state $0 \ldots 100 \ldots 0$ one time step ahead is to the state $0 \ldots 010 \ldots 0$ the probability of this transition is one, i.e.,

$$
P_{0 \ldots 010 \ldots 0}^{(n)}=1 \cdot P_{0 \ldots 100 \ldots 0}^{(n-1)}
$$

- $P_{00 \ldots 0}^{(n)}$ :

In this case we also have two possible states at the previous time instance, $00 \ldots 0$ and $00 \ldots 1$. Both these states are legal and both transitions are legal. From the state $00 \ldots 1$ there is only one possible transition, to the state $00 \ldots 0$, so the probability of this transition is one. From the state $00 \ldots 0$, two transitions are possible, to $00 \ldots 0$ and to $10 \ldots 0$. The latter transition has a probability of $\frac{1}{1+\Delta M}$ according to the discussion in the first point above. This means that the first transition has a probability of $1-\frac{\Delta M}{1+\Delta M}=\frac{\Delta M}{1+\Delta M}$, i.e.,

$$
P_{00 \ldots 0}^{(n)}=\frac{\Delta M}{1+\Delta M} P_{00 \ldots 0}^{(n-1)}+1 \cdot P_{00 \ldots 1}^{(n-1)} .
$$

To summarize, we have a transition probability from time difference $n-1$ to time difference $n$ of

$$
P^{(n)}=\underbrace{\left[\begin{array}{ccccc}
0 & 0 & \cdots & 0 & \frac{1}{1+\Delta M} \\
1 & 0 & \cdots & 0 & 0 \\
0 & 1 & \cdots & 0 & 0 \\
\vdots & \vdots & \ddots & \vdots & \vdots \\
0 & 0 & \cdots & 1 & \frac{\Delta M}{1+\Delta M}
\end{array}\right]}_{A} P^{(n-1)} .
$$

The assumption at the derivation of these probabilities was that $n \geq M-1$. However, we will see that this is true for any $n>0$. First consider $n=0$. Then we know that $X_{k+n}=X_{k}$, i.e.,

$$
P^{(0)}=\left[\begin{array}{c}
1 \\
0 \\
\vdots \\
0
\end{array}\right]
$$

For $0<n<M-1$, we have $P_{10 \ldots 0}^{(n)}=P_{00 \ldots 0}^{(n)}=0$ and

$$
P_{a_{1} a_{2} \ldots a_{M-1}}^{(n)}=\left\{\begin{array}{ll}
1 & \text { if } a_{n}=1, a_{i}=0, i \neq n \\
0 & \text { otherwise }
\end{array},\right.
$$

which is exactly

$$
P^{(n)}=A P^{(n-1)} .
$$


To calculate the covariance function we need the probability $P\left(X_{k+n}=X_{k}\right)$ which is equal to $P_{10 \ldots 0}^{(n)}$. This probability can be calculated recursively, for $n \geq 0$, by the state space form

$$
\begin{aligned}
& P^{(n+1)}=A P^{(n)}+B \delta[n+1] \\
& P\left(X_{k+n}=X_{k}\right)=C P^{(n)}
\end{aligned}
$$

where $A$ is as defined in (16) and

$$
\begin{aligned}
B & =\left[\begin{array}{llll}
1 & 0 & \cdots & 0
\end{array}\right]^{T} \\
C & =\left[\begin{array}{llll}
1 & 0 & \cdots & 0
\end{array}\right] .
\end{aligned}
$$

Here the driving impulse $\delta[n+1]$ is used instead of an initial state on $P$. The state space form only gives the probabilities for $n \geq 0$ but the probability is symmetric in time, so that

$$
P\left(X_{k-n}=X_{k}\right)=P\left(X_{k+n}=X_{k}\right) .
$$

\section{B. Covariance functions}

In this subsection we will evaluate the different parts (1114) of the covariance function $\bar{R}_{y}[n]$.

We start with the gain error covariance, $R_{\Delta_{g}}[n]$. The probabilities are the same, independent of the ADC number $i$, which gives

$$
\begin{aligned}
& R_{\Delta_{g}}[n]=E\left\{\left(1+\Delta_{g, X_{k+n}}^{0}\right)\left(1+\Delta_{g, X_{k}}^{0}\right)\right\} \\
& =P\left(X_{k+n}=X_{k}\right) \frac{1}{M+\Delta M} \sum_{i=0}^{M+\Delta M-1}\left(1+\Delta_{g, i}^{0}\right)^{2} \\
& +\left(1-P\left(X_{k+n}=X_{k}\right)\right) \frac{1}{(M+\Delta M-1)(M+\Delta M)} \\
& \sum_{i=0}^{M+\Delta M-1} \sum_{j \neq i}\left(1+\Delta_{g, i}^{0}\right)\left(1+\Delta_{g, j}^{0}\right) .
\end{aligned}
$$

From (17) we can calculate that

$$
\lim _{n \rightarrow \infty} P\left(X_{k+n}=X_{k}\right)=\frac{1}{M+\Delta M} .
$$

This means that $R_{\Delta_{g}}[n]$ does not converge to zero. We therefore rearrange (18) in one part, $\tilde{R}_{\Delta}$, that converge to zero and a constant.

$$
R_{\Delta_{g}}[n]=\alpha_{\Delta_{g}} \tilde{R}_{\Delta}[n]+\beta_{\Delta_{g}}
$$

where

$$
\begin{aligned}
& \tilde{R}_{\Delta}[n]=\left(P\left(X_{k+n}=X_{k}\right)-\frac{1}{M+\Delta M}\right), \\
& \alpha_{\Delta_{g}}=\frac{1}{M+\Delta M-1}\left(\sum_{i=0}^{M+\Delta M-1}\left(1+\Delta_{g, i}^{0}\right)^{2}\right. \\
& \left.-\frac{1}{M+\Delta M}\left(\sum_{i=0}^{M+\Delta M-1}\left(1+\Delta_{g, i}^{0}\right)\right)^{2}\right)
\end{aligned}
$$

and

$$
\beta_{\Delta_{g}}=\frac{1}{(M+\Delta M)^{2}}\left(\sum_{i=0}^{M+\Delta M-1}\left(1+\Delta_{g, i}^{0}\right)\right)^{2} .
$$

The offset error covariance, $R_{\Delta_{o}}[n]$, can be calculated in a similar way as the gain error covariance function

$$
\begin{aligned}
& R_{\Delta_{o}}[n]=E\left\{\Delta_{o, X_{k+n}}^{0} \Delta_{o, X_{k}}^{0}\right\} \\
& =\alpha_{\Delta_{o}} \tilde{R}_{\Delta}[n]+\beta_{\Delta_{o}}
\end{aligned}
$$

where

$$
\begin{aligned}
& \alpha_{\Delta_{o}}=\frac{1}{M+\Delta M-1}\left(\sum_{i=0}^{M+\Delta M-1}\left(\Delta_{o, i}^{0}\right)^{2}\right. \\
& \left.-\frac{1}{M+\Delta M}\left(\sum_{i=0}^{M+\Delta M-1} \Delta_{o, i}^{0}\right)^{2}\right)
\end{aligned}
$$

and

$$
\beta_{\Delta_{o}}=\frac{1}{(M+\Delta M)^{2}}\left(\sum_{i=0}^{M+\Delta M-1} \Delta_{o, i}^{0}\right)^{2} .
$$

To express the combined time error and signal covariance function, $R_{u, \Delta_{t}}[n]$, we have to involve the continuous time covariance function (6). We assume here that $R_{u}[n]=R_{u}(n)$, i.e., that the discrete covariance function is a sampled version of the continuous covariance function.

$$
\begin{aligned}
& R_{u, \Delta_{t}}[n]= \\
& \lim _{N \rightarrow \infty} \frac{1}{N} \sum_{n=1}^{N} E\left\{u\left(k+n+\Delta_{t, X_{k+n}}^{0}\right) u\left(k+\Delta_{t, X_{k}}^{0}\right)\right\} \\
& =P\left(X_{k+n}=X_{k}\right) R_{u}(n)+\left\{1-P\left(X_{k+n}=X_{k}\right)\right\} \\
& \frac{1}{(M+\Delta M-1)(M+\Delta M)} \\
& \sum_{i=0}^{M+\Delta M-1} \sum_{j \neq i} R_{u}\left(n+\Delta_{g, i}-\Delta_{g, j}\right) .
\end{aligned}
$$

This can be rearranged into one part that depends on the probabilistic model (17), and one part that does not

$$
\begin{aligned}
& R_{u, \Delta_{t}}[n]=\tilde{R}_{\Delta}[n]\left(R_{u}[n]-\tilde{R}_{u, \Delta_{t}}\right) \\
& +\frac{1}{M+\Delta M} R_{u}[n]+\frac{M+\Delta M-1}{M+\Delta M} \tilde{R}_{u, \Delta_{t}}[n]
\end{aligned}
$$

where

$$
\begin{gathered}
\tilde{R}_{u, \Delta_{t}}=\frac{1}{(M+\Delta M-1)(M+\Delta M)} . \\
\sum_{i=0}^{M+\Delta M-1} \sum_{j \neq i} R_{u}\left(n+\Delta_{t, i}-\Delta_{t, j}\right) .
\end{gathered}
$$

Finally, we should calculate the quantization noise part of the covariance function. With sufficiently many quantization levels, a uniformly distributed white noise is a good model of the quantization noise [5] for most input signals.

$$
R_{q}[n]=\sigma_{q}^{2} \delta[n]
$$

where $\sigma_{q}^{2}=\frac{q^{2}}{12}$, [6], and $q$ is the quantization step. 


\section{Spectrum}

The spectrum of a product of covariance functions is a convolution of the respective spectra [7]. This means that we can calculate the spectrum of $y[k]$ from $(15)$ as

$$
\begin{aligned}
\Phi_{y}\left(e^{i \omega}\right) & =\frac{1}{2 \pi} \int_{-\pi}^{\pi} \Phi_{u, \Delta_{t}}\left(e^{i(\omega-\gamma)}\right) \Phi_{\Delta_{g}}\left(e^{i \gamma}\right) d \gamma \\
& +\Phi_{\Delta_{o}}\left(e^{i \omega}\right)+\Phi_{q}\left(e^{i \omega}\right) .
\end{aligned}
$$

We will next evaluate each part of (27) separately, starting with $\Phi_{\Delta_{g}}\left(e^{i \omega}\right)$. From the definition of spectrum, we get

$$
\Phi_{\Delta_{g}}\left(e^{i \omega}\right)=\alpha_{\Delta_{g}} \sum_{n=-\infty}^{\infty} \tilde{R}_{\Delta}[n] e^{-i \omega n}+\beta_{\Delta_{g}} \sum_{n=-\infty}^{\infty} e^{-i \omega n}
$$

The second term of (28) evaluates to a Dirac function, if we restrict the domain to $\omega \in[-\pi, \pi]$.

$$
\beta_{\Delta_{g}} \sum_{n=-\infty}^{\infty} e^{-i \omega n}=2 \pi \beta_{\Delta_{g}} \delta(\omega), \omega \in[-\pi, \pi]
$$

Next, the first term of (28) will be evaluated. To evaluate this, we need to transform the state space description (17) to a transfer function

$$
\begin{aligned}
& \text { For } n \geq 0 \\
& P\left(X_{k+n}=X_{k}\right)=C(q I-A)^{-1} B q \delta[n] \\
& =\frac{q^{M-1}\left(q-\frac{\Delta M}{1+\Delta M}\right)}{q^{M-1}\left(q-\frac{\Delta M}{1+\Delta M}\right)-\frac{1}{1+\Delta M}} \delta[n] .
\end{aligned}
$$

The probability is symmetric so $P\left(X_{k+n}=X_{k}\right)=$ $P\left(X_{k-n}=X_{k}\right)$. In the same way we can write the constant part as output from a system

$$
\begin{aligned}
& \text { For } n \geq 0 \\
& \frac{1}{M+\Delta M}=\frac{1}{M+\Delta M} \frac{q}{q-1} \delta[n]
\end{aligned}
$$

Putting (30) and (31) together and eliminating the pole and zero in $q=1$ we get

For $n \geq 0$

$\tilde{R}_{\Delta}[n]=P\left(X_{k+n}=X_{k}\right)-\frac{1}{M+\Delta M}$

$=\zeta(M, \Delta M) \frac{q^{M-1}+\eta(M, \Delta M)\left((M-2) q^{M-2}+\cdots+q\right)}{q^{M-1}+\frac{1}{1+\Delta M}\left(q^{M-2}+\cdots+1\right)} \delta[n]$

where

$\zeta(M, \Delta M)=\frac{M+\Delta M-1}{M+\Delta M}$

and

$\eta(M, \Delta M)=\frac{1}{M-1+M \Delta M+(\Delta M)^{2}}$.
This means that we can calculate the spectrum as

$$
\begin{aligned}
& \tilde{\Phi}_{\Delta}\left(e^{i \omega}\right)=\sum_{n=-\infty}^{\infty} \tilde{R}_{\Delta}[n] e^{-i \omega n} \\
& =-\tilde{R}_{\Delta}[0]+2 \operatorname{Re}\left\{\sum_{n=0}^{\infty} \tilde{R}_{\Delta}[n] e^{-i \omega n}\right\}=-\zeta(M, \Delta M) \\
& +2 \operatorname{Re}\left\{\zeta \frac{e^{i \omega(M-1)}+\eta\left((M-2) e^{i \omega(M-2)}+\cdots+e^{i \omega}\right)}{e^{i \omega(M-1)}+\frac{1}{1+\Delta M}\left(e^{i \omega(M-2)}+\cdots+1\right)}\right\} .
\end{aligned}
$$

In the last expression the dependence on $M$ and $\Delta M$ is omitted for $\eta$ and $\zeta$ for convenience. In Fig. $6 \tilde{\Phi}_{\Delta}\left(e^{i \omega}\right)$ is shown for $M=8$ and $\Delta M=1,4,8$. This plot shows that the oscillations decrease when the number of additional ADCs is increased. This is expected since higher value of $\Delta M$ means that there are more ADCs to choose from at each sampling instance, and the errors are then more randomized. To summarize, we have

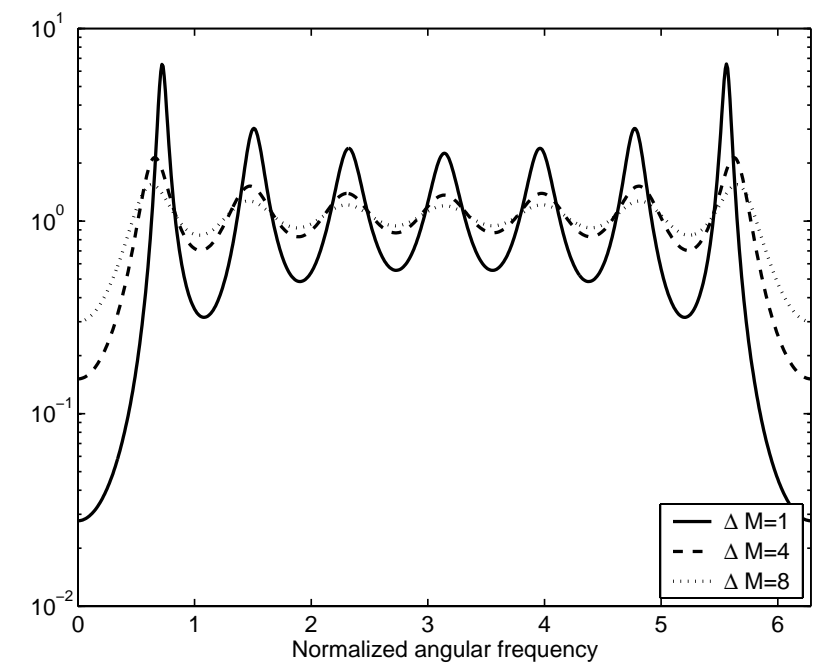

Fig. 6. The mismatch noise spectrum $\tilde{\Phi}_{\Delta}\left(e^{i \omega}\right)$ for $M=8$ and $\Delta M=$ $1,4,8$.

$$
\Phi_{\Delta_{g}}\left(e^{i \omega}\right)=\alpha_{\Delta_{g}} \tilde{\Phi}_{\Delta}\left(e^{i \omega}\right)+2 \pi \beta_{\Delta_{g}} \delta(\omega),
$$

where $\tilde{\Phi}_{\Delta}\left(e^{i \omega}\right)$ is as defined in (32). The offset error covariance (23) is similar to the gain error covariance (19) except for the constants $\alpha$ and $\beta$. This means that we get the offset error spectrum directly from (33) by replacing the constants

$$
\Phi_{\Delta_{o}}\left(e^{i \omega}\right)=\alpha_{\Delta_{o}} \tilde{\Phi}_{\Delta}\left(e^{i \omega}\right)+2 \pi \beta_{\Delta_{o}} \delta(\omega) .
$$

Next, the time error part of the spectrum will be evaluated. Calculating the spectrum from (24) we get

$$
\begin{aligned}
& \Phi_{u, \Delta_{t}}\left(e^{i \omega}\right) \\
& =\frac{1}{M+\Delta M} \Phi_{u}\left(e^{i \omega}\right)+\frac{M+\Delta M-1}{M+\Delta M} \tilde{\Phi}_{u, \Delta_{t}}\left(e^{i \omega}\right) \\
& +\frac{1}{2 \pi} \int_{-\pi}^{\pi} \tilde{\Phi}_{\Delta}\left(e^{i(\omega-\gamma)}\right)\left(\Phi_{u}\left(e^{i \gamma}\right)-\tilde{\Phi}_{u, \Delta_{t}}\left(e^{i \gamma}\right)\right) d \gamma .
\end{aligned}
$$

We have $\tilde{\Phi}_{\Delta}\left(e^{i \omega}\right)$ from (32), and $\Phi_{u}\left(e^{i \omega}\right)$ is the spectrum of the input signal. What remains to calculate then is $\tilde{\Phi}_{u, \Delta_{t}}\left(e^{i \omega}\right)$. 
To calculate this we have to start from the continuous time covariance function. If

$$
\tilde{R}(n)=R(n+\Delta),
$$

we have the spectrum [7]

$$
\tilde{\Phi}(\omega)=\Phi(\omega) e^{i \omega \Delta} .
$$

Using (36) in (25) we get

$$
\begin{array}{r}
\tilde{\Phi}_{u, \Delta_{t}}(\omega)=\frac{\Phi_{u}(\omega)}{(M+\Delta M-1)(M+\Delta M)} . \\
\sum_{k=0}^{M+\Delta M-1} \sum_{j \neq i} e^{i \omega\left(\Delta_{t, k}-\Delta_{t, j}\right)} .
\end{array}
$$

This can be rewritten as

$$
\begin{aligned}
& \tilde{\Phi}_{u, \Delta_{t}}(\omega)=-\Phi_{u}(\omega)+\frac{\Phi_{u}(\omega)}{(M+\Delta M-1)(M+\Delta M)} . \\
& \sum_{i=0}^{M+\Delta M-1} \sum_{j=0}^{M+\Delta M-1} \cos \left(\omega\left(\Delta_{t, i}-\Delta_{t, j}\right)\right) .
\end{aligned}
$$

The discrete time spectrum can be calculated from the continuous time spectrum using Poisson's summation formula [7]. Since we assume that $u(t)$ is band limited to the Nyquist frequency we have

$$
\tilde{\Phi}_{u, \Delta_{t}}\left(e^{i \omega}\right)=\tilde{\Phi}_{u, \Delta_{t}}(\omega), \omega \in[-\pi, \pi]
$$

Putting (38) and (32) into (35) we get

$$
\begin{aligned}
\Phi_{u, \Delta_{t}}\left(e^{i \omega}\right) & =\frac{1}{\zeta(M, \Delta M)}\left(\tilde{\Phi}_{\Delta} *\left[\Phi_{u} \cdot\left(1-H_{\Delta_{t}}\right)\right]\right)\left(e^{i \omega}\right) \\
& +\Phi_{u}\left(e^{i \omega}\right) H_{\Delta_{t}}(\omega),
\end{aligned}
$$

where $*$ denotes convolution, and

$$
\begin{aligned}
& H_{\Delta_{t}}(\omega)= \\
& \frac{1}{(M+\Delta M)^{2}} \sum_{i=0}^{M+\Delta M-1} \sum_{j=0}^{M+\Delta M-1} \cos \left(\omega\left(\Delta_{t, i}-\Delta_{t, j}\right)\right) .
\end{aligned}
$$

Finally, the quantization noise part of (27) should be evaluated. Here we have assumed a white noise model of the quantization, and the spectrum is therefore constant

$$
\Phi_{q}\left(e^{i \omega}\right)=\sigma_{q}^{2}
$$

To summarize, the output spectrum of the randomly interleaved ADC system is

$$
\begin{aligned}
\Phi_{y}\left(e^{i \omega}\right) & =\beta_{\Delta_{g}} \Phi_{u}\left(e^{i \omega}\right) H_{\Delta_{t}}(\omega) \\
& +\alpha_{\Delta_{g}}\left[\tilde{\Phi}_{\Delta} *\left(\Phi_{u} \cdot H_{\Delta_{t}}\right)\right]\left(e^{i \omega}\right) \\
& +\frac{\alpha_{\Delta_{g}}}{\zeta(M, \Delta M)}\left[\tilde{\Phi}_{\Delta} * \tilde{\Phi}_{\Delta} *\left(\Phi_{u} \cdot\left(1-H_{\Delta_{t}}\right)\right)\right]\left(e^{i \omega}\right) \\
& +\frac{\beta_{\Delta_{g}}}{\zeta(M, \Delta M)}\left[\tilde{\Phi}_{\Delta} *\left(\Phi_{u} \cdot\left(1-H_{\Delta_{t}}\right)\right)\right]\left(e^{i \omega}\right) \\
& +\alpha_{\Delta_{o}} \tilde{\Phi}_{\Delta}\left(e^{i \omega}\right)+2 \pi \beta_{\Delta_{o}} \delta(\omega)+\sigma_{q}^{2} .
\end{aligned}
$$

Here the first term is the signal part of the spectrum, and the rest is noise and distortion. We can see that even the signal part is somewhat distorted, by multiplication with $H_{\Delta_{t}}(\omega)$. However, this is not significant for most applications.

\section{Asymptotic properties}

If we in (32) let $\Delta M \rightarrow \infty$, while $M$ is kept constant we get

$$
\tilde{\Phi}_{\Delta}\left(e^{i \omega}\right)=1
$$

i.e., white noise. This is natural since we then can choose randomly from almost all ADCs at every time instance. Putting this into (43) the output spectrum is

$$
\begin{aligned}
\Phi_{y}\left(e^{i \omega}\right) & =\beta_{\Delta_{g}} \Phi_{u}\left(e^{i \omega}\right) H_{\Delta_{t}}(\omega) \\
& +\alpha_{\Delta_{g}} \frac{1}{2 \pi} \int_{-\pi}^{\pi} \Phi_{u}\left(e^{i \omega}\right) d \omega \\
& +\beta_{\Delta_{g}} \frac{1}{2 \pi} \int_{-\pi}^{\pi} \Phi_{u}\left(e^{i \omega}\right)(1-H(\omega)) d \omega \\
& +\alpha_{\Delta_{o}}+2 \pi \beta_{\Delta_{o}} \delta(\omega)+\sigma_{q}^{2} .
\end{aligned}
$$

The spectrum here consists of the signal spectrum, a Dirac pulse in $\omega=0$ and white noise, where the variance of the white noise depends on the variance of the gain, offset and time errors and the quantization.

\section{E. Sinusoidal input}

So far we have not assumed anything about the input signal. In this section we will evaluate the spectrum with a sinusoidal input and compare it with the case with no additional ADCs, i.e., $\Delta M=0$. We assume, in this section, an input signal

$$
u(t)=A \cos \left(\omega_{0} t\right) .
$$

The spectrum of $u(t)$ is then

$$
\Phi_{u}(\omega)=\frac{2 \pi A^{2}}{4}\left(\delta\left(\omega-\omega_{0}\right)+\delta\left(\omega+\omega_{0}\right)\right) .
$$

We assume that $A$ is chosen such that the full range of the ADC is used here. With an $N$-bit ADC, the quantization step then is $q=\frac{2 A}{2^{N}}$ and the quantization noise variance is $\sigma_{q}^{2}=$ $\frac{4 A^{2}}{12 \cdot 2^{2 N}}$. With random interleaving we then get the output signal spectrum by putting (44) into (43)

$$
\begin{aligned}
& \Phi_{y}\left(e^{i \omega}\right)=\frac{\pi A^{2} \beta_{\Delta_{g}}}{2} H_{\Delta_{t}}\left(\omega_{0}\right)\left(\delta\left(\omega-\omega_{0}\right)+\delta\left(\omega+\omega_{0}\right)\right) \\
& +\frac{A^{2} \alpha_{\Delta_{g}}}{4} H_{\Delta_{t}}\left(\omega_{0}\right)\left(\tilde{\Phi}_{\Delta}\left(e^{i\left(\omega-\omega_{0}\right)}\right)+\tilde{\Phi}_{\Delta}\left(e^{i\left(\omega+\omega_{0}\right)}\right)\right) \\
& +\frac{A^{2} \alpha_{\Delta_{g}}}{4 \zeta(M, \Delta M)}\left(1-H_{\Delta_{t}}\left(\omega_{0}\right)\right) . \\
& \left(\tilde{\Phi}_{\Delta} * \tilde{\Phi}_{\Delta}\left(e^{i\left(\omega-\omega_{0}\right)}\right)+\tilde{\Phi}_{\Delta} * \tilde{\Phi}_{\Delta}\left(e^{i\left(\omega+\omega_{0}\right)}\right)\right) \\
& +\frac{A^{2} \beta_{\Delta_{g}}}{4 \zeta(M, \Delta M)}\left(1-H_{\Delta_{t}}\left(\omega_{0}\right)\right) . \\
& \left(\tilde{\Phi}_{\Delta}\left(e^{i\left(\omega-\omega_{0}\right)}\right)+\tilde{\Phi}_{\Delta}\left(e^{i\left(\omega+\omega_{0}\right)}\right)\right) \\
& +\alpha_{\Delta_{o}} \tilde{\Phi}_{\Delta}\left(e^{i \omega}\right)+2 \pi \beta_{\Delta_{o}} \delta(\omega)+\frac{4 A^{2}}{12 \cdot 2^{2 N}} .
\end{aligned}
$$

Here we can see that the sinusoidal spectrum is in the first term, while the rest is noise and distortion. In Fig. 7 an example of a output signal spectrum from a randomly interleaved ADC with sinusoidal input is shown. 


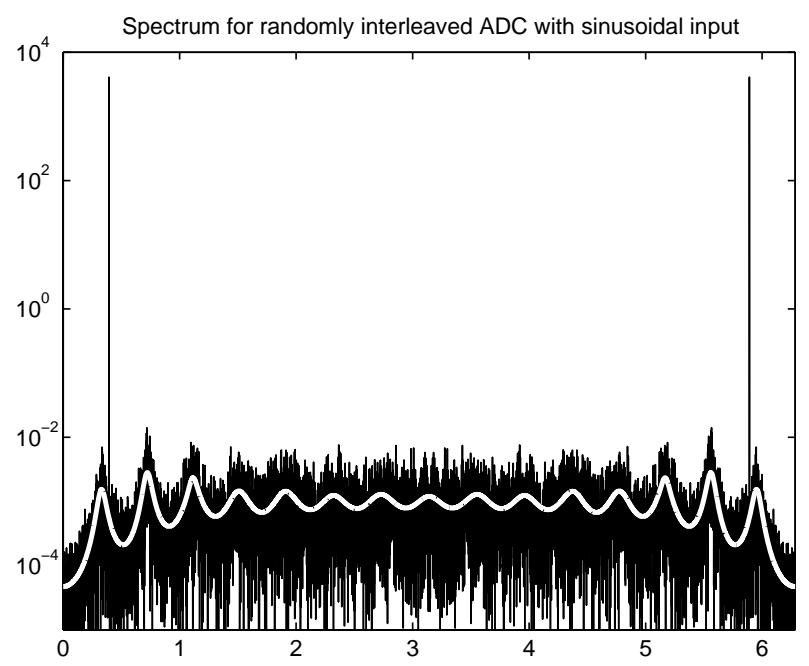

Fig. 7. Output signal spectrum from a randomly interleaved ADC system with sinusoidal input. Here $M=8$ and $\Delta M=1$. The black curve is the simulated spectrum and the white curve is the theoretical spectrum.

1) Fixed interleaving: For comparison, we will here evaluate the spectrum for a fixed interleaved ADC system $(\Delta M=$ 0 ) with sinusoidal input. The covariance function of $y[k]$ is as before

$$
R_{y}[n]=R_{\Delta_{g}}[n] R_{u, \Delta_{t}}[n]+R_{\Delta_{o}}[n]+R_{q}[n]
$$

which gives the spectrum

$$
\Phi_{y}\left(e^{i \omega}\right)=\Phi_{\Delta_{g}} * \Phi_{u, \Delta_{t}}\left(e^{i \omega}\right)+\Phi_{\Delta_{o}}\left(e^{i \omega}\right)+\Phi_{q}\left(e^{i \omega}\right) .
$$

We start with $R_{\Delta_{g}}[n]$, which is now periodic and symmetric

$$
\begin{aligned}
& R_{\Delta_{g}}[n]=\frac{1}{M} \sum_{i=0}^{M-1}\left(1+\Delta_{g, i}^{0}\right)\left(1+\Delta_{g,(i-n)}^{0} \bmod M\right) \\
& R_{\Delta_{g}}[M+n]=R_{\Delta_{g}}[n] \\
& R_{\Delta_{g}}[-n]=R_{\Delta_{g}}[n] .
\end{aligned}
$$

The spectrum is then given by [4]

$$
\Phi_{\Delta_{g}}\left(e^{i \omega}\right)=\frac{2 \pi}{M} \sum_{k=-M / 2}^{M / 2-1} \Phi_{\Delta_{g}}^{p}\left(e^{i 2 \pi k / M}\right) \delta(\omega-2 \pi k / M),
$$

where

$$
\Phi_{\Delta_{g}}^{p}\left(e^{i \omega}\right)=\sum_{n=0}^{M-1} R_{\Delta_{g}}[n] e^{-i \omega n} .
$$

The calculations for $R_{\Delta_{o}}[n]$ are similar and we get the covariance function

$$
R_{\Delta_{o}}[n]=\frac{1}{M} \sum_{i=0}^{M-1}\left(\Delta_{o, i}^{0} \Delta_{o,(i-n)}^{0} \bmod M\right)
$$

and the spectrum

$$
\Phi_{\Delta_{o}}\left(e^{i \omega}\right)=\frac{2 \pi}{M} \sum_{k=-M / 2}^{M / 2-1} \Phi_{\Delta_{o}}^{p}\left(e^{i 2 \pi k / M}\right) \delta(\omega-2 \pi k / M),
$$

where

$$
\Phi_{\Delta_{o}}^{p}\left(e^{i \omega}\right)=\sum_{n=0}^{M-1} R_{\Delta_{o}}[n] e^{-i \omega n}
$$

The above expressions are valid for a general input signal. However, for the time error part we restrict the calculations to a sinusoidal input for notational simplicity. The time error covariance function is

$$
\begin{aligned}
& R_{u, \Delta_{t}}[n] \\
& =A^{2} \lim _{N \rightarrow \infty} \frac{1}{N} \sum_{k=0}^{N-1} \cos \left(k+n+\Delta_{t, k+n}\right) \cos \left(k+\Delta_{t, k}\right) \\
& =\frac{A^{2}}{2} \lim _{N \rightarrow \infty} \frac{1}{N} \sum_{k=0}^{N-1}\left(\cos \left(2 \omega_{0} k+\omega_{0} n+\omega_{0}\left(\Delta_{t, k+n}+\Delta_{t, k}\right)\right)\right. \\
& \left.+\cos \left(\omega_{0} n+\omega_{0}\left(\Delta_{t, k+n}-\Delta_{t, k}\right)\right)\right) \\
& =\frac{A^{2}}{2 M} \sum_{k=0}^{M-1} \cos \left(\omega_{0} n+\omega_{0}\left(\Delta_{t, k+n}-\Delta_{t, k}\right)\right)
\end{aligned}
$$

Calculating the Fourier transform of (50) we get the spectrum

$$
\begin{aligned}
& \Phi_{u, \Delta_{t}}\left(e^{i \omega}\right)=\frac{\pi A^{2}}{M} \sum_{k=-M / 2}^{M / 2-1} \Phi_{\Delta_{t}}^{p}\left(e^{i 2 \pi k / M}\right) . \\
& {\left[\delta\left(\omega-\left(\omega_{0}+\frac{2 \pi k}{M}\right)_{[-\pi, \pi]}\right)\right.} \\
& \left.+\delta\left(\omega+\left(\omega_{0}-\frac{2 \pi k}{M}\right)_{[-\pi, \pi]}\right)\right] .
\end{aligned}
$$

where

$$
\Phi_{\Delta_{t}}^{p}\left(e^{i \omega}\right)=\sum_{n=0}^{M-1} R_{\Delta_{t}}[n] e^{i \omega n}
$$

and

$$
R_{\Delta_{t}}[n]=\frac{1}{M} \sum_{i=0}^{M-1} \cos \left(\omega_{0}\left(\Delta_{t, i+n}-\Delta_{t, i}\right)\right) .
$$

Here $\omega_{[-\pi, \pi]}=\omega+n * 2 \pi$ where $n$ is an integer such that $\omega_{[-\pi, \pi]} \in[-\pi, \pi]$. Putting (48), (49) and (51) into (47) we get the output spectrum for the fixed interleaved ADC system

$$
\begin{aligned}
& \Phi_{y}\left(e^{i \omega}\right)= \\
& \frac{\pi A^{2}}{2 M^{2}} \sum_{m=-M / 2}^{M / 2-1} \sum_{k=-M / 2}^{M / 2-1} \Phi_{\Delta_{g}}^{p}\left(e^{i \frac{2 \pi m}{M}}\right) \Phi_{u, \Delta_{t}}^{p}\left(e^{i \frac{2 \pi k}{M}}\right) . \\
& {\left[\delta\left(\omega-\left(\omega_{0}+\frac{2 \pi(k+m)}{M}\right)_{[-\pi, \pi]}\right)\right.} \\
& \left.+\delta\left(\omega+\left(\omega_{0}+\frac{2 \pi(k+m)}{M}\right)_{[-\pi, \pi]}\right)\right] \\
& +\frac{2 \pi}{M} \sum_{k=-M / 2}^{M / 2-1} \Phi_{\Delta_{o}}^{p}\left(e^{i 2 \pi k / M}\right) \delta(\omega-2 \pi k / M) \\
& +\frac{4 A^{2}}{12 \cdot 2^{2 N}} .
\end{aligned}
$$


An example of the output spectrum from a fixed interleaved ADC system with sinusoidal input and mismatch errors was shown in Fig. 2.

2) SNDR: In this section we will calculate and compare the SNDR for random interleaved ADCs and fixed interleaved ADCs. For the randomly interleaved ADC the signal energy is

$$
\begin{aligned}
& E\left\{s^{2}[k]\right\} \\
& =\frac{2 \pi A^{2} \beta_{\Delta_{g}}}{4} H_{\Delta_{t}}\left(\omega_{0}\right) \int_{-\pi}^{\pi}\left(\delta\left(\omega-\omega_{0}\right)+\delta\left(\omega+\omega_{0}\right)\right) d \omega \\
& =\pi A^{2} \beta_{\Delta_{g}} H_{\Delta_{t}}\left(\omega_{0}\right) .
\end{aligned}
$$

We get the distortion energy by integrating all but the first term of (45)

$$
\begin{aligned}
& E\left\{e^{2}[k]\right\}=\pi A^{2} \alpha_{\Delta_{g}} \zeta(M, \Delta M)+\pi A^{2} \beta_{\Delta_{g}}\left(1-H_{\Delta_{t}}\left(\omega_{0}\right)\right) \\
& +2 \pi \zeta(M, \Delta M) \alpha_{\Delta_{o}}+2 \pi \beta_{\Delta_{o}}+\frac{2 \pi A^{2}}{3 \cdot 2^{2 N}}
\end{aligned}
$$

If we assume that the mean values of the respective errors are zero, we get

$$
\begin{aligned}
& \beta_{\Delta_{o}}=0, \beta_{\Delta_{g}}=1 \\
& \alpha_{\Delta_{o}}=\frac{1}{\zeta(M, \Delta M)} \sigma_{\Delta_{o}}^{2}, \alpha_{\Delta_{g}}=\frac{1}{\zeta(M, \Delta M)} \sigma_{\Delta_{g}}^{2},
\end{aligned}
$$

where

$$
\begin{aligned}
\sigma_{\Delta_{o}}^{2} & =\frac{1}{M+\Delta M} \sum_{i=0}^{M+\Delta M-1} \Delta_{o, i}^{2} \\
\sigma_{\Delta_{g}}^{2} & =\frac{1}{M+\Delta M} \sum_{i=0}^{M+\Delta M-1} \Delta_{g, i}^{2} .
\end{aligned}
$$

Further, if we assume that the time errors are small $H_{\Delta_{t}}(\omega)$ can be approximated by a Taylor expansion

$$
H_{\Delta_{t}}(\omega) \approx 1-\omega^{2} \sigma_{\Delta_{t}}^{2}
$$

where

$$
\sigma_{\Delta_{t}}^{2}=\frac{1}{M+\Delta M} \sum_{i=0}^{M+\Delta M-1} \Delta_{t, i}^{2}
$$

With these assumptions (52) can be simplified to

$$
E\left\{e^{2}[k]\right\}=\pi A^{2}\left(\sigma_{\Delta_{g}}^{2}+\omega_{0}^{2} \sigma_{\Delta_{t}}^{2}+\frac{\sigma_{\Delta_{o}}^{2}}{A^{2} / 2}+\frac{2}{3 \cdot 2^{2 N}}\right) .
$$

With the assumption that the mean values of the errors are zero and that the time errors are small we get the signal energy for the fixed interleaved case

$$
\begin{aligned}
& E\left\{s^{2}[k]\right\}=\frac{\pi A^{2}}{M^{2}} \sum_{k=-M / 2}^{M / 2-1} \Phi_{\Delta_{g}}^{p}\left(e^{i \frac{2 \pi k}{M}}\right) \Phi_{\Delta_{t}}^{p}\left(e^{i \frac{2 \pi k}{M}}\right) \\
& \approx \pi A^{2}\left(1-\omega_{0}^{2} \sigma_{\Delta_{t}}^{2}\right)
\end{aligned}
$$

and the distortion energy

$$
\begin{aligned}
& E\left\{e^{2}[k]\right\}=\int_{-\pi}^{\pi} \Phi_{y}\left(e^{i \omega}\right) d \omega-E\left(s^{2}[k]\right) \\
& \approx \pi A^{2}\left(\sigma_{\Delta_{g}}^{2}+\omega_{0}^{2} \sigma_{\Delta_{t}}^{2}+\frac{\sigma_{\Delta_{o}}^{2}}{A^{2} / 2}+\frac{2}{3 \cdot 2^{2 N}}\right),
\end{aligned}
$$

which is exactly the same as for the randomly interleaved case.

This means that the SNDR is the same for both the randomly interleaved ADC system and the fixed interleaved ADC system

$$
\begin{aligned}
& S N D R= \\
& 10 \log _{10}\left(\frac{\pi A^{2}\left(1-\omega_{0}^{2} \sigma_{\Delta_{t}}^{2}\right)}{\pi A^{2}\left(\sigma_{\Delta_{g}}^{2}+\omega_{0}^{2} \sigma_{\Delta_{t}}^{2}+\frac{\sigma_{\Delta_{o}}^{2}}{A^{2} / 2}+\frac{2}{3 \cdot 2^{2 N}}\right)}\right) \\
& \approx-10 \log _{10}\left(\sigma_{\Delta_{g}}^{2}+\omega_{0}^{2} \sigma_{\Delta_{t}}^{2}+\frac{\sigma_{\Delta_{o}}^{2}}{A^{2} / 2}+\frac{2}{3 \cdot 2^{2 N}}\right) .
\end{aligned}
$$

This is expected since we cannot change the total amount of distortion by changing the order in which we select the ADCs. However, the shape of the distortion is very different between the fixed interleaved and the randomly interleaved case. If we study the SFDR we can see that in the randomly interleaved case there are no $\delta$-spikes in the output spectrum. This means that the SFDR is infinite in the randomly interleaved case.

3) Noise comparison: Since we have quantization noise in the ADC, we do not gain much performance by decreasing the mismatch error noise below a certain level for the randomly interleaved ADC system. This level depends on the number of bits in the ADC. In this subsection we will compare the noise caused by the mismatch errors with the quantization noise. The comparison can be done in two ways. The first way is to compare the mean noise, i.e., the SNDR. The other way is to compare the maximum value of spectra. We will evaluate both cases here. We have the SNDR an $N$-bit ADC system from (54)

$$
S N D R \approx-10 \log _{10}\left(\sigma_{\Delta_{g}}^{2}+\omega_{0}^{2} \sigma_{\Delta_{t}}^{2}+\frac{\sigma_{\Delta_{o}}^{2}}{A^{2} / 2}+\frac{2}{3 \cdot 2^{2 N}}\right)
$$

If we compare the quantization noise part with the mismatch noise part and calculate a limit such that the mismatch error noise contributes less than the quantization noise we get

$$
\sigma_{\Delta_{g}}^{2}+\omega_{0}^{2} \sigma_{\Delta_{t}}^{2}+\frac{\sigma_{\Delta_{o}}^{2}}{A^{2} / 2}<\frac{2}{3} 4^{-N}
$$

To give a bound for each type of error we can assume that each part in (55) contributes equally much and further more put $\omega_{0}=\pi$ to get an upper bound on the time error part

$$
\begin{aligned}
\sigma_{\Delta_{g}}^{2} & <\frac{2}{9} 4^{-N} \\
\sigma_{\Delta_{t}}^{2} & <\frac{2}{9 \pi^{2}} 4^{-N} \\
\sigma_{\Delta_{o}}^{2} & <\frac{A^{2}}{9} 4^{-N} .
\end{aligned}
$$

If we instead consider the maximum value of the spectrum, the maximum of the noise part of (45) should be smaller than the quantization noise. The maximum value depends on the input signal frequency, therefore we maximize over both $\omega$ 
and $\omega_{0}$.

$$
\begin{aligned}
& \max _{\omega, \omega_{0}}\left\{\frac{A^{2} \alpha_{\Delta_{g}}}{4} H_{\Delta_{t}}\left(\omega_{0}\right)\left(\tilde{\Phi}_{\Delta}\left(e^{i\left(\omega-\omega_{0}\right)}\right)+\tilde{\Phi}_{\Delta}\left(e^{i\left(\omega+\omega_{0}\right)}\right)\right)\right. \\
& +\frac{A^{2} \alpha_{\Delta_{g}}}{4 \zeta(M, \Delta M)}\left(1-H_{\Delta_{t}}\left(\omega_{0}\right)\right) . \\
& \left(\tilde{\Phi}_{\Delta} * \tilde{\Phi}_{\Delta}\left(e^{i\left(\omega-\omega_{0}\right)}\right)+\tilde{\Phi}_{\Delta} * \tilde{\Phi}_{\Delta}\left(e^{i\left(\omega+\omega_{0}\right)}\right)\right) \\
& +\frac{A^{2} \beta_{\Delta_{g}}}{4 \zeta(M, \Delta M)}\left(1-H_{\Delta_{t}}\left(\omega_{0}\right)\right) . \\
& \left(\tilde{\Phi}_{\Delta}\left(e^{i\left(\omega-\omega_{0}\right)}\right)+\tilde{\Phi}_{\Delta}\left(e^{i\left(\omega+\omega_{0}\right)}\right)\right) \\
& \left.+\alpha_{\Delta_{o}} \tilde{\Phi}_{\Delta}\left(e^{i \omega}\right)\right\}<\frac{4 A^{2}}{12 \cdot 2^{2 N}} .
\end{aligned}
$$

If we again split the mismatch errors into three equal parts, we get

$$
\begin{aligned}
\sigma_{\Delta_{g}}^{2} & <\frac{1}{\max _{\omega} \tilde{\Phi}_{\Delta}\left(e^{i \omega}\right)} \frac{2 \zeta(M, \Delta M)}{9} 4^{-N} \\
\sigma_{\Delta_{t}}^{2} & <\frac{1}{\max _{\omega} \tilde{\Phi}_{\Delta}\left(e^{i \omega}\right)} \frac{2 \zeta(M, \Delta M)}{9 \pi^{2}} 4^{-N} \\
\sigma_{\Delta_{o}}^{2} & <\frac{1}{\max _{\omega} \tilde{\Phi}_{\Delta}\left(e^{i \omega}\right)} \frac{A^{2} \zeta(M, \Delta M)}{9} 4^{-N} .
\end{aligned}
$$

It is hard to find a general analytical solution to $\max _{\omega} \tilde{\Phi}_{\Delta}\left(e^{i \omega}\right)$, but for $M=2$ the maximum is

$$
\max _{\omega} \tilde{\Phi}_{\Delta}\left(e^{i \omega}\right)=\zeta(M, \Delta M)\left(1+\frac{2}{\Delta M}\right) .
$$

Putting (62) into (59-61) the upper bounds for the errors are

$$
\begin{aligned}
\sigma_{\Delta_{g}}^{2} & <\frac{\Delta M}{\Delta M+2} \frac{2}{9} 4^{-N} \\
\sigma_{\Delta_{t}}^{2} & <\frac{\Delta M}{\Delta M+2} \frac{2}{9 \pi^{2}} 4^{-N} \\
\sigma_{\Delta_{o}}^{2} & <\frac{\Delta M}{\Delta M+2} \frac{A^{2}}{9} 4^{-N} .
\end{aligned}
$$

In Fig. 8 the dependence of $\frac{1}{\zeta(M, \Delta M)} \max _{\omega} \tilde{\Phi}_{\Delta}\left(e^{i \omega}\right)$ on $M$ is shown for some different values of $\Delta M$. Fig. 9 shows the dependence of $\frac{1}{\zeta(M, \Delta M)} \max _{\omega} \tilde{\Phi}_{\Delta}\left(e^{i \omega}\right)$ on $\Delta M$ for some different values of $M$.

\section{MISMATCH ERROR ESTIMATION}

If the offset errors are known, the output signal can be corrected from the offset errors by subtracting the offset from the respective subsequences

$$
z_{i}^{\left(\Delta_{o}^{0}\right)}\left[k_{i}\right]=y_{i}\left[k_{i}\right]-\Delta_{o, i}^{0}
$$

If the gain errors are known, the offset corrected signal can be further corrected with the gain errors

$$
z_{i}^{\left(\Delta_{o}^{0}, \Delta_{g}^{0}\right)}\left[k_{i}\right]=\frac{1}{1+\Delta_{g, i}^{0}} z_{i}^{\left(\Delta_{o}^{0}\right)}\left[k_{i}\right] .
$$

Assuming that the input signal is quasi-stationary and zero mean, we get

$$
\bar{m}_{y_{i}}=\Delta_{o, i}^{0} \text {. }
$$

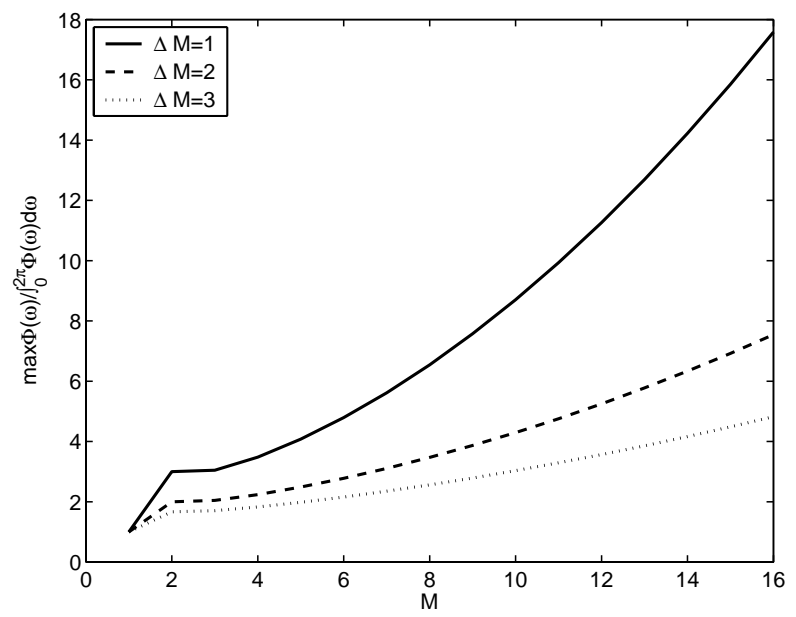

Fig. 8. $\frac{1}{\zeta(M, \Delta M)} \max _{\omega} \tilde{\Phi}_{\Delta}\left(e^{i \omega}\right)$ is plotted as a function of $M$ for $\Delta M=$ $1,2,3$. The maximum value increase with $M$ as expected since there are relatively fewer ADCs to choose from at each sample instance.

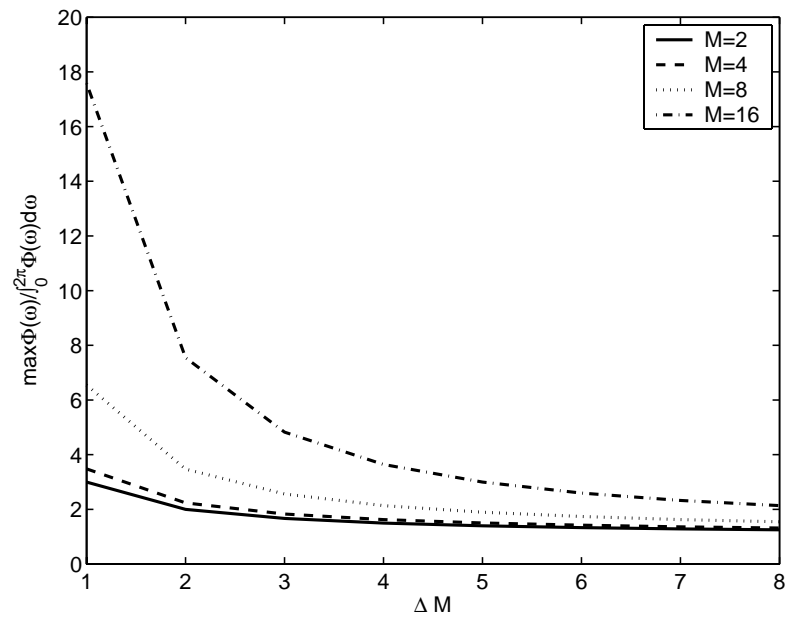

Fig. 9. $\frac{1}{\zeta(M, \Delta M)} \max _{\omega} \tilde{\Phi}_{\Delta}\left(e^{i \omega}\right)$ is plotted as a function of $\Delta M$ for $M=2,4,8,16$. The maximum value decreases with $\Delta M$ as expected since there are more ADCs to choose from at each sample instance.

This means that we can estimate the offset error by

$$
\begin{aligned}
& \text { For } i=0, \ldots, M+\Delta M-1 \\
& \hat{\Delta}_{o, i}=\frac{1}{N_{i}} \sum_{k_{i}} y_{i}\left[k_{i}\right]
\end{aligned}
$$

where $N_{i}$ is the number of samples in the sequence $y_{i}$.

The gain errors can be estimated by taking the mean absolute value of each offset corrected subsequence

$$
\begin{aligned}
& \text { For } i=0, \ldots, M+\Delta M-1 \\
& \hat{\Delta}_{g, i}=\frac{\frac{1}{N_{i}} \sum_{k_{i}}\left|z_{i}^{\left(\hat{\Delta}_{o}\right)}\left[k_{i}\right]\right|}{\frac{1}{M+\Delta M} \sum_{j=0}^{M+\Delta M-1}\left\{\frac{1}{N_{j}} \sum_{k_{j}}\left|z_{j}^{\left(\hat{\Delta}_{o}\right)}\left[k_{j}\right]\right|\right\}}-1 .
\end{aligned}
$$

The time errors are harder to estimate since that requires that we study the difference between samples, and the samples are not taken in the same order here. However, if the gain and 
offset errors are dominating it can still be useful to estimate the gain and offset.

Assume that the samples of $y\left[k_{i}\right]$ are independent and identically distributed. This is not really true, but since the time instances for the subsequences are picked at random it is a rather good approximation. Under these assumptions the mean values are approximately Gaussian distributed according to the central limit theorem, if $N_{i}$ is large enough

$$
\hat{\Delta}_{o, i}=\hat{m}_{y_{i}}=\frac{1}{N_{i}} \sum_{k_{i}} y\left[k_{i}\right] \in \mathrm{N}\left(\Delta_{o, i}, \frac{\sigma_{u}^{2}}{N_{i}}\left(1+\Delta_{g, i}^{0}\right)^{2}\right)
$$

and

$$
\begin{aligned}
& \hat{m}_{\left|z_{i}^{\left(\hat{\Delta}_{o}\right)}\right|}= \\
& \frac{1}{N_{i}} \sum_{k_{i}}\left|z_{i}^{\left(\hat{\Delta}_{o}\right)}\left[k_{i}\right]\right| \in \mathrm{N}\left(\left(1+\Delta_{g, i}^{0}\right) m_{|u|}, \frac{\sigma_{|u|}^{2}}{N_{i}}\left(1+\Delta_{g, i}^{0}\right)^{2}\right) .
\end{aligned}
$$

The distribution of (65) is actually only valid if $\hat{\Delta}_{o}=\Delta_{o}^{0}$, but it is approximately valid with small deviations from the true value. The denominator of (63) also has an approximately Gaussian distribution. But since the same value is used for the estimation of all $\Delta_{g, i}$, an error in this value, just gives a bias to the all gain error estimates and does not affect the gain error noise spectrum. This means that we can treat it as a constant, and we get from (65)

$$
\hat{\Delta}_{g, i} \in \mathrm{N}\left(\Delta_{g, i}^{0}, \frac{\sigma_{|u|}^{2}}{m_{|u|}^{2} N_{i}}\left(1+\Delta_{g, i}^{0}\right)^{2}\right) .
$$

If we assume that the gain errors are small we can simplify (64) and (66) to

$$
\hat{\Delta}_{o, i} \in \mathrm{N}\left(\Delta_{o, i}, \frac{\sigma_{u}^{2}}{N_{i}}\right)
$$

and

$$
\hat{\Delta}_{g, i} \in \mathrm{N}\left(\Delta_{g, i}^{0}, \frac{\sigma_{|u|}^{2}}{m_{|u|}^{2} N_{i}}\right) .
$$

Furthermore, a sum of squares of $N(0,1)$-distributed variables is $\chi^{2}$-distributed with $M-1+\Delta M$ degrees of freedom [8].

$$
\sum_{i=0}^{M-1+\Delta M} \frac{\left(\hat{\Delta}_{o, i}-\Delta_{o, i}^{0}\right)^{2}}{\sigma_{u} / \sqrt{N_{i}}} \in \chi^{2}(M-1+\Delta M)
$$

and

$$
\sum_{i=0}^{M-1+\Delta M} \frac{\left(\hat{\Delta}_{g, i}-\Delta_{g, i}^{0}\right)^{2}}{\sigma_{|u|} / m_{|u|} \sqrt{N_{i}}} \in \chi^{2}(M-1+\Delta M) .
$$

If we assume that $N_{i} \approx \tilde{N}$ for all $i$, we can make confidence intervals for $\bar{\sigma}_{\Delta_{o}}^{2}$ and $\bar{\sigma}_{\Delta_{g}}^{2}$

and

$$
\bar{\sigma}_{\Delta_{o}}^{2}<\frac{\sigma_{u}^{2}}{\tilde{N}} \frac{\chi_{\alpha}^{2}(M+\Delta M-1)}{M+\Delta M}
$$

$$
\bar{\sigma}_{\Delta_{g}}^{2}<\frac{\sigma_{|u|}^{2}}{m_{|u|}^{2} \tilde{N}} \frac{\chi_{\alpha}^{2}(M+\Delta M-1)}{M+\Delta M}
$$

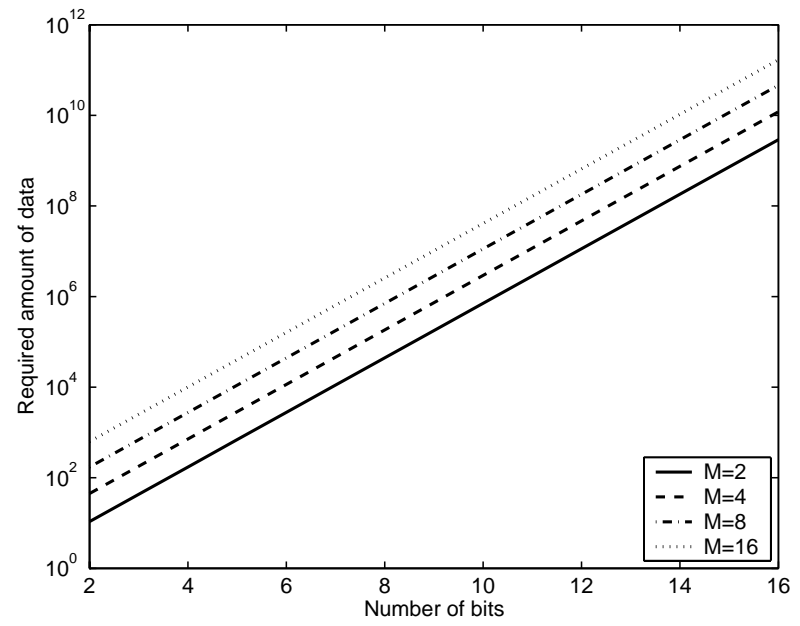

Fig. 10. Required amount of estimation data to get the offset mismatch noise below the quantization noise. The confidence level is here 0.95 and $\Delta M=1$.

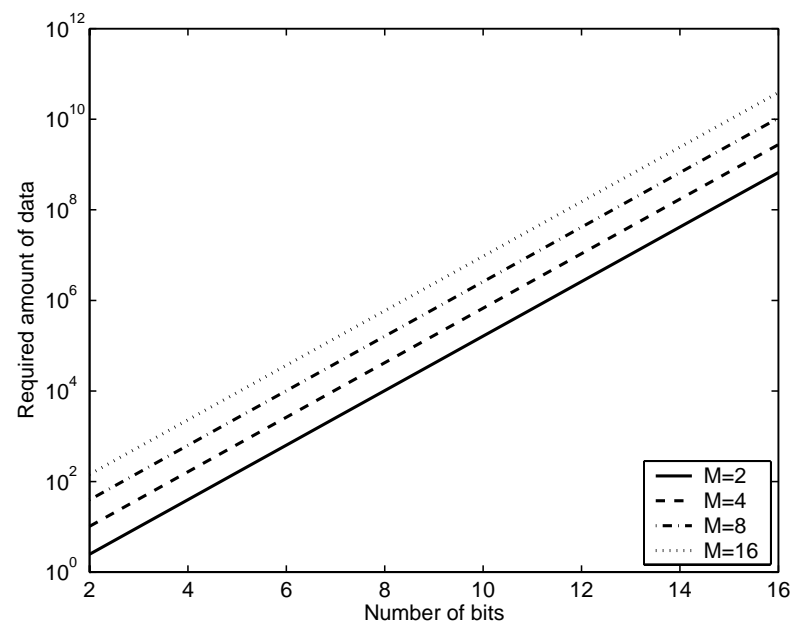

Fig. 11. Required amount of estimation data to get the gain mismatch noise below the quantization noise. The confidence level is here 0.95 and $\Delta M=1$.

where $1-\alpha$ is the confidence level. This means that to achieve the mean bounds for an $N$-bit ADC, (56) and (58), we need

$$
\tilde{N}>\frac{\sigma_{u}^{2}}{A^{2}} \frac{\chi_{\alpha}^{2}(M+\Delta M-1)}{M+\Delta M} 9 \cdot 4^{N}
$$

for the offset error estimates and

$$
\tilde{N}>\frac{\sigma_{|u|}^{2}}{m_{|u|}^{2}} \frac{\chi_{\alpha}^{2}(M+\Delta M-1)}{M+\Delta M} \frac{9}{2} 4^{N}
$$

for the gain error estimates. Similarly, to achieve the max bounds, (59) and (61), we need

$$
\tilde{N}>\frac{\sigma_{u}^{2}}{A^{2}} \frac{\chi_{\alpha}^{2}(M+\Delta M-1)}{M+\Delta M-1} 9 \max _{\omega} \tilde{\Phi}_{\Delta}(\omega) 4^{N}
$$

for the offset error estimates and

$$
\tilde{N}>\frac{\sigma_{|u|}^{2}}{m_{|u|}^{2}} \frac{\chi_{\alpha}^{2}(M+\Delta M-1)}{M+\Delta M-1} \frac{9 \max _{\omega} \tilde{\Phi}_{\Delta}(\omega)}{2} 4^{N}
$$


for the gain error estimates. In Fig. 10 and 11, the required amount of data to estimate offset and gain are shown for varying number of bits, i.e., equations (69) and (70). In these figures the confidence level is 0.95 and $\Delta M=1$. The plots are here generated for sinusoidal input, but the results are similar with other input signals.

\section{Measurements}

Measurements were done on a 12-bit randomly interleaved ADC with $M=16$ and $\Delta M=1$. First, the randomization was turned off and only 16 ADCs were used. This is shown in Fig. 12, where the input is sinusoidal. Here we see that the interleaving mismatch cause a lot of distortion. In Fig. 13 the randomization is used. Here the distortion peaks are eliminated and we see a spectrum similar to the theoretical spectrum shown in Fig. 7. In this example the randomization improves the SFDR with about $20 \mathrm{~dB}$.

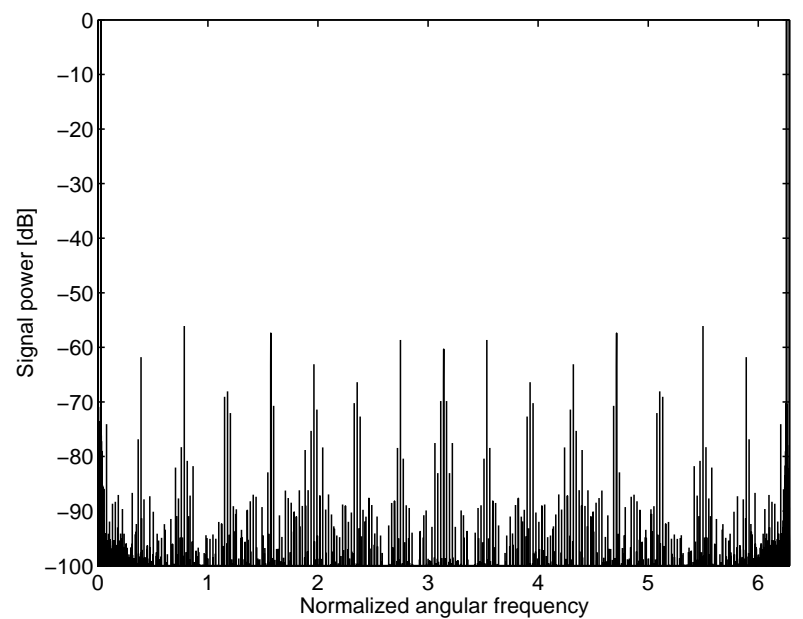

Fig. 12. Output spectrum from a time interleaved ADC without randomization.

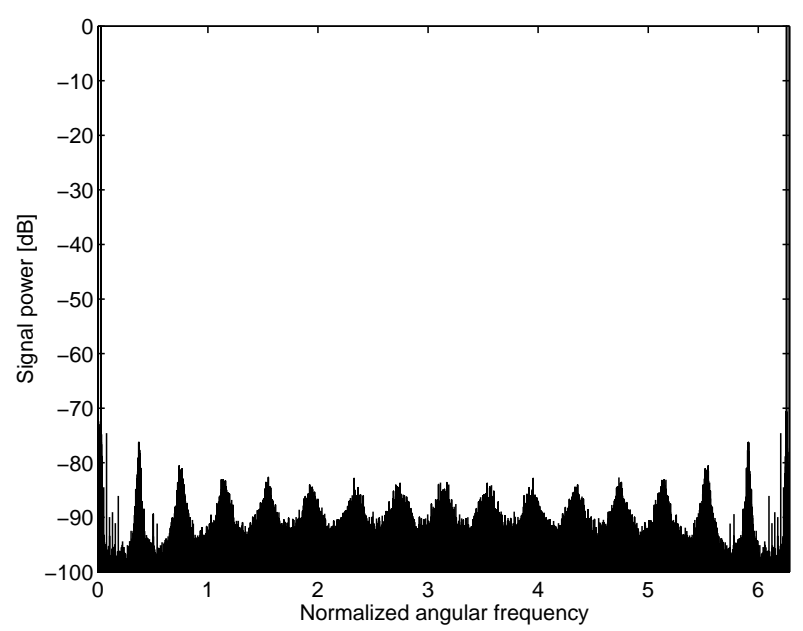

Fig. 13. Output spectrum from a randomly interleaved ADC system with $M=16$ and $\Delta M=1$.

\section{CONCLUSION}

To achieve significantly higher sampling rates in A/D conversion a time interleaved ADC system is a good option. However, due to the manufacturing process all the ADCs in the time interleaved system are not identical. This means that mismatch errors in time, gain and offset are introduced into the system. The mismatch errors cause distortion in the sampled signal, which severely decrease the SFDR.

One way to decrease the impact of the mismatch errors, is to randomize the order in which the ADCs are used. Additional ADCs are then used in the interleaved ADC system to enable two or several ADCs to select from at each sampling instance. By doing this the mismatch distortion is transformed to a more noise-like shape. In this paper we have studied the randomly interleaved ADC system from a probabilistic viewpoint. In Section III we have presented a probabilistic model for the ADC system and derived the spectrum caused by mismatch errors. We have also calculated how small the errors must be to give less noise contribution than the quantization for a given number of bits. In Section IV we have discussed how the offset and gain errors can be estimated and compensated for. We have also calculated how much estimation data that is required to get the mismatch noise below the quantization noise.

\section{REFERENCES}

[1] Y.-C. Jenq, "Digital spectra of nonuniformly sampled signals: A robust sampling time offset estimation algorithm for ultra high-speed waveform digitizers using interleaving," IEEE Transactions on Instrumentation and Measurement, vol. 39, no. 1, pp. 71-75, February 1990.

[2] J. Elbornsson and J.-E. Eklund, "Blind estimation of timing errors in interleaved AD converters," in Proc. ICASSP 2001, vol. 6. IEEE, 2001, pp. 3913-3916.

[3] M. Tamba, A. Shimizu, H. Munakata, and T. Komuro, "A method to improve SFDR with random interleaved sampling method," in ITC International Test Conference, 2001, pp. 512-520.

[4] L. Ljung, System Identification, Theory for the user, 2nd ed. PrenticeHall, 1999.

[5] B. Widrow, I. Kollar, and M.-C. Liu, "Statistical theory of quantization," IEEE Transactions on Instrumentation and Measurement, vol. 45, no. 2, pp. 353-361, April 1996.

[6] R. van de Plassche, Integrated Analog-to-Digital and Digital-to-Analog Converters. Kluwer Academic Publishers, 1994.

[7] M. Hayes, Statistical digital signal processing and modeling. Wiley, 1996.

[8] G. Blom, Sannolikhetslära med tillämpningar. Studentlitteratur, 1984, in Swedish. 